\title{
A Stochastic String with a Compound Poisson Process
}

\author{
Sheng Fan \\ Department of Mathematics, Southwestern University of Finance and Economics, Chengdu 611130, China \\ Correspondence should be addressed to Sheng Fan; fansheng@swufe.edu.cn
}

Received 28 May 2013; Accepted 10 July 2013

Academic Editor: Shaoyong Lai

Copyright (C) 2013 Sheng Fan. This is an open access article distributed under the Creative Commons Attribution License, which permits unrestricted use, distribution, and reproduction in any medium, provided the original work is properly cited.

We investigate a compound Poisson infinite factor diffusion model which describes the relationship between the infinite-dimension random risk resource and the corresponding stochastic process. We derive the no-arbitrage condition on the drift of instantaneous forward rates in the compound model and study the impact of random jump on the price of the zero-coupon bond.

\section{Introduction}

In the study of the stochastic process, the expansion from single-factor model to multiple-factor model greatly improves its capability to describe the dynamic properties. For the term structure of interest rates, multiple-factor expansion enables itself to more flexibly describe the relationship between different maturities and their corresponding risk-free interest rates. The term structure curve contains rich information about the economy status and financial markets. The literatures have witted extensive researches on this topic for recent decades. For instance, the HJM model in $[1,2]$ provides insight about arbitrage-free condition of the shift and volatility of the dynamic process of forward interest rates under the risk-free condition. Kennedy [3, 4], Goldstein [5], and Santa-Clara and Didier [6] model each time-to-maturity forward rate driven by its own shock termed Brown Sheet, Random Field, or Stochastic String. In fact, the models in [3-6] expand the multiple-factor model to infinite stochastic factor with imperfect correlation among different maturities. At the same time, the correlations are not confined by the function form of volatilities. These results display greater variability in the correlation between different maturities.

In this paper, according to the stochastic string model presented in [6], we introduce a compound Poisson process with a constant jump intensity and random jump size to capture information burst and resulting discontinuous path. We derive the no-arbitrage condition in the compound Poisson infinite-dimensional diffusion process in which the drift and volatility of stochastic process under the risk-neutral measure are satisfied. Some of the results in [6] are extended.

The paper is organized as follows. Section 2 reviews the single-factor, multiple-factor model and stochastic string model, which are related to our study. In Section 3, we add a random jump process in the stochastic string model discussed in [6] and analyze its dynamic properties. An application of our main result is presented in Section 4.

\section{Single-Factor, Multiple-Factor Model and Stochastic String Model}

For a probability space $(\Omega, \Phi, P)$, the dynamics of a stochastic process $f(t, x)$ is described by

$$
d f(t, x)=\alpha(t, x) d t+\sigma(t, x) d W(t),
$$

where $W(t)$ is a standard Wiener process and $\alpha(t, x)$ and $\sigma(t, x)$ are drift and volatility terms, respectively. When $f(t, x)$ is explained as the instantaneous forward rate at time $t$ for time-to-maturity $x>0$, (1) is the HJM model. The price at time $t$ of a bond with maturity $s$ is

$$
B(t, s)=\exp \left[-\int_{0}^{s-t} f(t, x) d x\right] .
$$

The HJM model has no-arbitrage condition which is written by

$$
\alpha(t, x)=\frac{\partial f(t, x)}{\partial x}+\sigma(t, x)\left[\int_{0}^{x} \sigma(t, y) d y+\theta(t)\right],
$$


where the process $\theta(t)$ denotes the market price of risk at time $t$. Using Itô's formula, we calculate the dynamics of bond prices as

$$
\begin{aligned}
\frac{d B(t, s)}{B(t, s)}= & {\left[f(t, s-t)-\int_{0}^{s-t} \alpha(t, y) d y\right.} \\
& \left.+\frac{1}{2}\left(\int_{0}^{s-t} \sigma(t, y) d y\right)^{2}\right] d t \\
& -\left[\int_{0}^{s-t} \sigma(t, y) d y\right] d W(t) .
\end{aligned}
$$

Under the no-arbitrage condition, the instantaneous forward rate of time-to-maturity $x$ is

$$
\begin{aligned}
f(t, x)= & f(0, t+x)+\int_{0}^{t} \eta(u, t+x-u) d u \\
& +\int_{0}^{t} \sigma(u, t+x-u) d W(u),
\end{aligned}
$$

where

$$
\eta(t, x) \equiv \sigma(t, x)\left[\int_{0}^{x} \sigma(t, v) d v+\theta(t)\right] .
$$

In fact, every term structure model driven by Brownian motion is a special case of the HJM model (e.g., the Vasicek model in [7], the Hull-White model in [8], etc.). However, the single-factor HJM model has a limitation that different time-to-maturities are completely correlated; that is, their correlation coefficient equals 1 . This implies that

(1) the term structure curve would parallel shift which limits its capability to generate richer class of dynamics and shapes of the term structure of interest rates,

(2) it does not permit consistency with term structure innovation along with the time.

One way to extend the model is by introducing the $N$ dimensional Brownian motion to the term structure model written by

$$
d f(t, x)=\alpha(t, x) d t+\sum_{i=1}^{N} \sigma^{i}(t, x) d W^{i} .
$$

One can obtain the correlation of the forward rate curve driven by the $N$-dimensional Brownian motion as

$$
c(x, y)=\frac{\sum_{i=1}^{N} \sigma^{i}(t, x) \sigma^{i}(t, y)}{\sqrt{\sum_{i=1}^{N}\left(\sigma^{i}(t, x)\right)^{2}} \sqrt{\sum_{i=1}^{N}\left(\sigma^{i}(t, y)\right)^{2}}} .
$$

Different from the multiple-factor HJM model in (7), which implies all assets shocked by the common $N$ factors, SantaClara and Didier [6] model the forward rate as a stochastic string and allow each forward rate to be driven by its own random risk resource.

Assume that the dynamics of the instantaneous forward rates at $t$ with time-to-maturity $x(x>0)$ is modeled by

$$
d_{t} f(t, x)=\alpha(t, x) d t+\sigma(t, x) d_{t} W(t, x),
$$

where $W(t, x)$ is a generalization of Brownian motion which depends on both time $t$ and time-to-maturity $x$. The notation $d_{t}()$ means that the increment is taken with respect to time $t$. Equation (9) is not the infinite-dimensional simple generalization of the multifactor HJM model, in which all forward rates are subject to the same (possibly infinite) set of Brownian motion shocks. In this model, there is one different stochastic shock for each time-to-maturity. Given time-tomaturity $x$, the stochastic perturbation $W(t, x)$ is a standard Wiener process, which satisfies several requirements (see [6] for details). For example, the correlation coefficient matrix is positive semidefinite, which means that, for all functions $h(x)$ in $L^{2}$ and for some large enough constant $T$,

$$
\int_{0}^{T} \int_{0}^{T} h(x) c(x, y) h(y) d y d x \geq 0 .
$$

The no-arbitrage condition of the drift of instantaneous forward rate is

$$
\begin{aligned}
\alpha(t, x)= & \frac{\partial f(t, x)}{\partial x}+\sigma(t, x) \\
& \times\left[\int_{0}^{x} c(x, y) \sigma(t, y) d y+\int_{0}^{\infty} c(x, y) \theta(t, y) d y\right] .
\end{aligned}
$$

The solution of the stochastic differential equation for instantaneous forward interest rate is

$$
\begin{aligned}
f(t, x)= & f(0, t+x)+\int_{0}^{t} a(u, t+x-u) d u \\
& +\int_{0}^{t} \sigma(u, t+x-u) d_{u} W(u, t+x-u),
\end{aligned}
$$

where

$$
\begin{aligned}
a(t, x) \equiv & \sigma(t, x) \\
& \times\left[\int_{0}^{x} c(x, y) \sigma(t, y) d y+\int_{0}^{\infty} c(x, y) \theta(t, y) d y\right] .
\end{aligned}
$$

Under the arbitrage-free condition, we have

$$
\begin{aligned}
B(t, s)=B(0, s) \exp \left[\int_{0}^{t} r(u) d u-\int_{0}^{t} U(u, s-u) d u\right. \\
\left.\quad-\int_{0}^{t} V(u, s-u) d_{u} W(u, s-u)\right]
\end{aligned}
$$

where

$$
\begin{gathered}
U(t, x) \equiv \int_{0}^{\infty} \theta(t, y) \int_{0}^{x} c(y, z) \sigma(t, z) d z d y \\
V(t, x) \equiv \int_{0}^{x} \sigma(t, y) d y .
\end{gathered}
$$


The covariance of different time-to-maturity zero-coupon bond yields is

$$
\begin{aligned}
\operatorname{cov}[ & {\left[\frac{d B(t, s)}{B(t, s)}, \frac{d B\left(t, s^{\prime}\right)}{B\left(t, s^{\prime}\right)}\right] } \\
& =\int_{0}^{s-t} \sigma(t, x)\left(\int_{0}^{s^{\prime}-t} c(x, y) \sigma(t, y) d y\right) d x d t .
\end{aligned}
$$

\section{Compound Poisson Infinite-Dimensional Diffusion Process}

Information burst, some emergency events, crises, and monetary target adjustment may cause the return and price of asset jump. There are more and more empirical lines of evidence which show that the interest rate models should incorporate the jump risk. The difference of the asset price of continuous path will disappear as the change of time converges to zero. We know that in the jump model, the difference does not converge to zero, although its probability of jump occurrence will converge to 0. Eberlein et al. [9] investigate the term structure models driven by Lévy processes.

Based on the continuous path of instantaneous forward rate used in Santa-Clara and Didier [6] and other works [35], we introduce a compound Poisson process to capture the yield curve jump. For each time-to-maturity $x$, assume that the dynamics of instantaneous forward rate is

$$
d_{t} f(t, x)=\alpha(t, x) d t+\sigma(t, x) d_{t} W(t, x)+\gamma(t, x) d J(t),
$$

where $J(t)$ is a compound Poisson process and $J(t)=$ $\sum_{m=1}^{N(t)} Y_{m}$. Poisson process $N(t)$ counts the number of jumps that occur at or before time $t . Y_{m}(m=1,2, \ldots)$ are independent identically distributed discrete random variables with finite values $a_{1}, a_{2}, \ldots a_{K}$, with probability $P\left(y_{j}=a_{i}\right)=p_{i}$, $\sum_{i=1}^{K} p_{i}=1 \cdot \gamma(t, x)$ is a nonrandom function. The compound jump is independent of the stochastic string $W(t, x)$.

Applying the decomposition theorem of a compound Poisson process (see [10]), $J(t)=\sum_{m=1}^{N(t)} Y_{m}$ may be rewritten as $J(t)=\sum_{i=1}^{K} a_{i} d N_{i}(t)$. So, we can rewrite the dynamics of instantaneous forward rates as

$$
\begin{aligned}
f(t, x)= & f(0, x)+\int_{0}^{t} \alpha(u, x) d u+\int_{0}^{t} \sigma(u, x) d_{u} W(u, x) \\
& +\sum_{i=1}^{K} \int_{0}^{t} \gamma(u, x) a_{i} d N_{i}(u)
\end{aligned}
$$

where $W(t, x)$ is the same random risk resource as defined in (9).

Assume that the market is complete, so that the existence and uniqueness of the risk market price are guaranteed. It can be proved that the continuous part of random risk factor is independent of the jump risk factor.

For the continuous risk resource part, define

$$
\widetilde{W}(t, x)=W(t, x)+\int_{0}^{t} \int_{0}^{\infty} \theta(u, y) c(x, y) d y d u,
$$

or in differential form

$$
d_{t} \widetilde{W}(t, x)=d_{t} W(t, x)+\int_{0}^{\infty} \theta(t, y) c(x, y) d y d t .
$$

The correlation coefficient for different time-to-maturity random risk resource $\widetilde{W}(t, s)$ is the same as that of $W(t, s)$. For fixed time-to-maturity $x$, let

$$
\begin{aligned}
& Z_{0}(t, x) \\
& =\exp \left[-\int_{0}^{t} \int_{0}^{\infty} \theta(u, y) c(x, y) d y d_{u} W(u, y)\right. \\
& -\frac{1}{2} \int_{0}^{t} \int_{0}^{\infty}\left[\theta(u, z) c(x, z) \int_{0}^{\infty} \theta(u, y) c(x, y) d y\right] \\
& \quad \times c(y, z) d z d u], \\
& Z_{i}(t, x)=\exp \left[\left(\lambda_{i}-\widetilde{\lambda}_{i}\right) t+N_{i}(t) \log \left(\widetilde{\lambda}_{i}-\lambda_{i}\right)\right], \\
& Z(t, x)=\prod_{i=0}^{K} Z_{i}(t, x), \\
& \widetilde{P}(A)=\int_{A} Z(T, x) d P \quad \forall A \in \Phi ., \ldots, K,
\end{aligned}
$$

We obtain the risk-neutral measure $\widetilde{P}$ under some conditions. Using the properties of risk-neutral measure $\widetilde{P}$, for fixed timeto-maturity $x$, we know that $\widetilde{W}(t, x)$ is a martingale. At the same time, $J(t)=\sum_{i=1}^{K} a_{i} d N_{i}(t)$ is a compound Poisson process with intensity $\tilde{\lambda}$. The jump sizes $Y_{1}, Y_{2} \cdots$ are still I.I.D. random variables, while the jump intensity satisfies $\widetilde{P}\left(Y_{j}=\right.$ $\left.a_{i}\right)=\tilde{p}\left(a_{i}\right)=\left(\tilde{\lambda}_{i} / \tilde{\lambda}\right)(i=1,2, \ldots, K)$ for arbitrary $j$, where $\sum_{i=1}^{K} \widetilde{\lambda_{i}}=\tilde{\lambda}$ and each $\tilde{\lambda_{i}}(i=1,2, \ldots, K)$ is a positive constant. Notice that, under the new measure $\widetilde{P}, \widetilde{W}(t, x)$ is still independent of $N_{i}(t), i=1,2, \ldots, K$.

Theorem 1. If the market is complete, the arbitrage-free condition of the instantaneous forward rate satisfies

$$
\begin{aligned}
\alpha(t, x)= & \frac{\partial f(t, x)}{\partial x}+\sigma(t, x) \\
& \times\left[\int_{0}^{x} \sigma(t, y) c(x, y) d y+\int_{0}^{\infty} \theta(t, y) c(x, y) d y\right] \\
& -\sum_{i=1}^{K} a_{i} \widetilde{\lambda_{i}} \gamma(t, x) \exp \left[-a_{i} \int_{0}^{x} \gamma(t, y) d y\right] .
\end{aligned}
$$


Proof. The price at time $t$ of a zero-coupon bond with maturity $s$ is $B(t, s)=\exp \left[-\int_{0}^{s-t} f(t, x) d x\right]$. For fixed $s$, we have

$$
\ln (B(t, s))=-\int_{0}^{s-t} f(t, x) d x
$$

Differentiating with respect to time $t$ in (23), we obtain

$$
\begin{aligned}
d_{t} \ln B(t, s)= & f(t, s-t) d t-\int_{0}^{s-t} d_{t} f(t, x) d x \\
= & f(t, s-t) d t-\int_{0}^{s-t} \alpha(t, x) d x d t \\
& -\int_{0}^{s-t} \sigma(t, x) d_{t} W(t, x) d x \\
& -\int_{0}^{s-t} \gamma(t, x) \sum_{i=1}^{K} a_{i} d N_{i}(t) d x . \\
= & {\left[f(t, s-t)-\int_{0}^{s-t} \alpha(t, x) d x\right] d t } \\
& -\int_{0}^{s-t} \sigma(t, x) d x d_{t} W(t, x) \\
& -\sum_{i=1}^{K} \int_{0}^{s-t} \gamma(t, x) d x a_{i} d N_{i}(t) .
\end{aligned}
$$

Applying jump process Itô's formula (see [10]), we have

$$
\begin{aligned}
& d_{t} B(t, s) \\
& =B(t, s)\left[f(t, s-t)-\int_{0}^{s-t} \alpha(t, x) d x\right. \\
& \left.\quad+\frac{1}{2} \int_{0}^{s-t} \sigma(t, x) \int_{0}^{s-t} \sigma(t, y) c(x, y) d y d x\right] d t \\
& \quad-B(t, s) \int_{0}^{s-t} \sigma(t, x) d x d_{t} W(t, x) \\
& +B\left(t^{-}, s\right) \sum_{i=1}^{K}\left[\exp \left(-a_{i} \int_{0}^{s-t} \gamma(t, x) d x\right)-1\right] d N_{i}(t) .
\end{aligned}
$$

Under the risk-neutral probability measure $\widetilde{P}$, the price of zero-coupon bond satisfies

$$
\begin{aligned}
d_{t} B(t, s)= & B(t, s) r(t) d t \\
& -B(t, s) \int_{0}^{s-t} \sigma(t, x) d x d_{t} \widetilde{W}(t, x) \\
& +B\left(t^{-}, s\right) \sum_{i=1}^{K}\left[\exp \left(-a_{i} \int_{0}^{s-t} \gamma(t, x) d x\right)-1\right] \\
& \times d\left(N_{i}(t)-\widetilde{\lambda}_{i} t\right) .
\end{aligned}
$$

Combining (25) with (26), we have

$$
\begin{aligned}
r(t)= & f(t, s-t)-\int_{0}^{s-t} \alpha(t, x) d x \\
& +\frac{1}{2} \int_{0}^{s-t} \sigma(t, x) \int_{0}^{s-t} \sigma(t, y) c(x, y) d y d x \\
& +\int_{0}^{s-t} \sigma(t, x) \int_{0}^{\infty} \theta(t, y) c(x, y) d y d x \\
& +\sum_{i=1}^{K}\left[\exp \left(-a_{i} \int_{0}^{s-t} \gamma(t, x) d x\right)-1\right] \tilde{\lambda}_{i}
\end{aligned}
$$

which is the expression that the risk market prices satisfy. It includes market prices of $K$ jump risk factors and infinite random risk resource. Note that the compensated Poisson process $N_{i}(t)-\widetilde{\lambda}_{i} t$ in (26) is a martingale. Let $s-t=x$, and applying the stochastic Fubini theorem, we rewrite (27) as

$$
\begin{aligned}
r(t)= & f(t, x)-\int_{0}^{x} \alpha(t, y) d y \\
& +\frac{1}{2} \int_{0}^{x} \sigma(t, z) \int_{0}^{x} \sigma(t, y) c(z, y) d y d z \\
& +\int_{0}^{x} \sigma(t, z) \int_{0}^{\infty} \theta(t, y) c(z, y) d y d z \\
& +\sum_{i=1}^{K}\left[\exp \left(-a_{i} \int_{0}^{x} \gamma(t, y) d y\right)-1\right] \tilde{\lambda}_{i} .
\end{aligned}
$$

Differentiating (28) with respect to $x$, we obtain

$$
\begin{aligned}
\frac{\partial f(t, x)}{\partial x} & -\alpha(t, x)+\sigma(t, x) \\
\times & {\left[\int_{0}^{x} \sigma(t, y) c(x, y) d y+\int_{0}^{\infty} \theta(t, y) c(x, y) d y\right] } \\
& -\sum_{i=1}^{K} a_{i} \widetilde{\lambda_{i}} \gamma(t, x) \exp \left[-a_{i} \int_{0}^{x} \gamma(t, y) d y\right]=0 .
\end{aligned}
$$

Rearranging (29), we obtain the arbitrage-free condition of (22). It completes the proof.

Remark 2. When $a_{i}=0(i=1,2, \ldots K),(22)$ reduces to

$$
\begin{aligned}
\alpha(t, x)= & \frac{\partial f(t, x)}{\partial x}+\sigma(t, x) \int_{0}^{x} \sigma(t, y) c(x, y) d y \\
& +\sigma(t, x) \int_{0}^{\infty} \theta(t, y) c(x, y) d y
\end{aligned}
$$

which is the arbitrage-free condition presented in [6]. Moreover, for arbitrary time-to-maturity $x$ and $y$, if $c(x, y)=1$, and let $\int_{0}^{\infty} \theta(t, y) d y=\Theta(t)$, then the arbitrage-free condition becomes

$$
\alpha(t, x)=\frac{\partial f(t, x)}{\partial x}+\sigma(t, x)\left[\int_{0}^{x} \sigma(t, y) d y+\Theta(t)\right],
$$


which is the arbitrage-free condition of the one-dimensional HJM model.

Under the risk-neutral measure $\widetilde{P}$, the dynamics of instantaneous forward rates which satisfy the arbitrage-free condition can be rewritten as

$$
\begin{aligned}
d_{t} f(t, x)= & A(t, x) d t+\sigma(t, x) d_{t} \widetilde{W}(t, x) \\
& +\sum_{i=1}^{K} a_{i} \gamma(t, x) d N_{i}(t),
\end{aligned}
$$

where

$$
\begin{aligned}
A(t, x) \equiv & \frac{\partial f(t, x)}{\partial x}+\sigma(t, x) \int_{0}^{x} \sigma(t, y) c(x, y) d y \\
& -\sum_{i=1}^{K} a_{i} \widetilde{\lambda_{i}} \gamma(t, x) \exp \left[-a_{i} \int_{0}^{x} \gamma(t, y) d y\right] .
\end{aligned}
$$

Theorem 3. Under the risk-neutral measure $\widetilde{P}$, the price at time t of a zero-coupon bond with maturitys is

$$
\begin{aligned}
& B(t, s) \\
& =B(0, s) \\
& \quad \times \exp \left[\int_{0}^{t} r(u) d u-\int_{0}^{t} \int_{0}^{s-u} \sigma(u, x) d x d_{u} \widetilde{W}(u, x)\right. \\
& \quad-\frac{1}{2} \int_{0}^{t} d u \int_{0}^{s-u} \sigma(u, x) \\
& \quad \times \int_{0}^{s-u} \sigma(u, y) c(x, y) d y d x \\
& \left.\quad-\sum_{i=1}^{K} \int_{0}^{t}\left[\exp \left(-a_{i} \int_{0}^{s-u} \gamma(u, x) d x\right)-1\right] \widetilde{\lambda_{i}} d u\right] \\
& \quad \times \exp \left[-\sum_{i=1}^{K} \int_{0}^{t} \int_{0}^{s-u} \gamma(u, x) d x a_{i} d N_{i}(u)\right] .
\end{aligned}
$$

Proof. We show that (34) is the solution of (26). Obviously, when $t=0$, the result is correct. Let $Y(t)=B(0, s) \exp \left[X^{c}(t)\right]$, where

$$
\begin{aligned}
X^{c}(t) \equiv & \int_{0}^{t} r(u) d u-\int_{0}^{t} \int_{0}^{s-u} \sigma(u, x) d x d_{u} \widetilde{W}(u, x) \\
& -\frac{1}{2} \int_{0}^{t} d u \int_{0}^{s-u} \sigma(u, x) \int_{0}^{s-u} \sigma(u, y) c(x, y) d y d x \\
& -\sum_{i=1}^{K} \int_{0}^{t}\left[\exp \left(-a_{i} \int_{0}^{s-u} \gamma(u, x) d x\right)-1\right] \widetilde{\lambda_{i}} d u
\end{aligned}
$$

Denote

$$
K(t) \equiv \exp \left[-\sum_{i=1}^{K} \int_{0}^{t} \int_{0}^{s-u} \gamma(u, x) d x a_{i} d N_{i}(u)\right] .
$$

Then, we have

$$
\begin{aligned}
d Y(t)=Y(t)\left[r(t) d t-\int_{0}^{s-t} \sigma(t, x) d x d_{t} \widetilde{W}(t, x)\right. \\
\left.\quad-\sum_{i=1}^{K}\left[\exp \left(-a_{i} \int_{0}^{s-t} \gamma(t, x) d x\right)-1\right] \widetilde{\lambda_{i}} d t\right],
\end{aligned}
$$

$d K(t)=K\left(t^{-}\right)$

$$
\times\left[\sum_{i=1}^{K}\left[\exp \left(-a_{i} \int_{0}^{s-t} \gamma(t, x) d x\right)-1\right] d N_{i}(t)\right] .
$$

Notice that as the length of time interval converges to 0 , there is at most one jump in each time interval. Combining (37) with (38), we obtain

$$
\begin{aligned}
d_{t} B(t, s) & \\
= & Y(t) d K(t)+d Y(t) K(t)+d Y(t) d K(t) \\
= & Y(t) K\left(t^{-}\right)\left[\sum_{i=1}^{K}\left[\exp \left(-a_{i} \int_{0}^{s-t} \gamma(t, x) d x\right)-1\right] d N_{i}(t)\right] \\
& +Y(t) K(t)\left[r(t) d t-\int_{0}^{s-t} \sigma(t, x) d x d_{t} \widetilde{W}(t, x)\right. \\
& +0 \\
= & B\left(t^{-}, s\right) \sum_{i=1}^{K}\left[\exp \left(-a_{i} \int_{0}^{s-t} \gamma(t, x) d x\right)-1\right] \\
& \times d\left(N_{i}(t)-\widetilde{\lambda}_{i} t\right) \\
& +B(t, s)\left[r(t) d t-\int_{0}^{s-t} \sigma(t, x) d x d_{t} \widetilde{W}(t, x)\right] .
\end{aligned}
$$

Therefore, (34) is the solution of (26). It completes the proof.

Remark 4. In (34), it shows that the price of zero-coupon bond is affected by two parts: continuous component and jump component. For the continuous part, besides the riskfree interest rate effect, the stochastic string risk resources till to the maturity would generate an accumulated effect on the price of zero-coupon bond. As the occurrence of jump, its long-term accumulative effects will be offset by part of $-\sum_{i=1}^{K} \int_{0}^{t}\left[\exp \left(-a_{i} \int_{0}^{s-u} \gamma(u, x) d x\right)-1\right] \widetilde{\lambda_{i}} d u$. The correlation of different maturities from time $t$ to maturity $s$ has an explicit effect on the price. For the jump component, the effect is scaled by the function $\gamma(t, x)$, which captures the jump effect on the financial variables. 


\section{Application}

The compound Poisson infinite diffusion model developed in Section 3 comprises the traditional jump-diffusion model. We consider that the jump sizes are discrete random variables. It is convenient to introduce the continuous jump size model whose size is, for instance, normally distributed random variable.

We consider the price of the short-term interest rate futures. Assume that the financial market is shocked by two kinds of random risk resource: one is continuous risk factor of $W(t, x)$ and the other is a compound Poisson process. Assume that the market is complete. Consider a futures contract maturity at $T_{f}, t<T_{f}<s$, whose underlying asset is the zerocoupon bond maturity at $s$. Denote the futures price at time $t$ as $F\left(t, T_{f}, s\right)$. Under the risk-neutral measure $\widetilde{P}$, the futures price $F\left(t, T_{f}, s\right)$ is a martingale. We have

$$
F\left(t, T_{f}, s\right)=E_{t}\left[F\left(T_{f}, T_{f}, s\right) \mid \Phi_{t}\right]=E_{t}^{\widetilde{P}}\left[B\left(T_{f}, s\right) \mid \Phi_{t}\right] .
$$

Theorem 5. Under the risk-neutral measure $\widetilde{P}$, the futures price with underlying asset as a zero-coupon bond is

$$
\begin{aligned}
& F\left(t, T_{f}, s\right) \\
& =F\left(0, T_{f}, s\right) \\
& \quad \times \exp \left[-\int_{0}^{t} \int_{T_{f}}^{s} \sigma(u, x-u) d x d_{u} \widetilde{W}(u, x-u)\right. \\
& \quad-\sum_{i=1}^{K} \int_{0}^{t} \int_{T_{f}}^{s} \gamma(u, x-u) d x a_{i} d N_{i}(u) \\
& \quad-\frac{1}{2} \int_{0}^{t}\left(\int_{T_{f}}^{s} \sigma(u, x-u) d x\right. \\
& \left.\quad+\sum_{i=1}^{K} \int_{0}^{t} \widetilde{\lambda_{i}}\left[\exp \left(-a_{i} \int_{T_{f}}^{s} \gamma(u, x-u) d x\right)-1\right] d u\right] .
\end{aligned}
$$

Proof. Note that the zero-coupon bond price is a function of instantaneous forward rates

$$
B\left(T_{f}, s\right)=\exp \left[-\int_{0}^{s-T_{f}} f\left(T_{f}, x\right) d x\right] .
$$

Therefore, we have

$$
\begin{aligned}
\ln & B\left(T_{f}, s\right) \\
& =-\int_{0}^{s-T_{f}} f\left(T_{f}, x\right) d x \\
& =-\int_{0}^{s-T_{f}} f\left(0, T_{f}+x\right) d x
\end{aligned}
$$

$$
\begin{aligned}
& -\int_{0}^{s-T_{f}} \int_{0}^{T_{f}} A\left(u, T_{f}+x-u\right) d u d x \\
& -\int_{0}^{s-T_{f}} \int_{0}^{T_{f}} \sigma\left(u, T_{f}+x-u\right) d_{u} \widetilde{W}\left(u, T_{f}+x-u\right) d x \\
& -\sum_{i=1}^{K} \int_{0}^{s-T_{f}} \int_{0}^{T_{f}} \gamma\left(u, T_{f}+x-u\right) a_{i} d N_{i}(u) d x \\
= & \int_{T_{f}}^{s} f(0, x) d x-\int_{T_{f}}^{s} \int_{0}^{T_{f}} A(u, x-u) d u d x \\
& -\int_{T_{f}}^{s} \int_{0}^{T_{f}} \sigma(u, x-u) d_{u} \widetilde{W}(u, x-u) d x \\
& -\sum_{i=1}^{K} \int_{T_{f}}^{s} \int_{0}^{T_{f}} \gamma(u, x-u) a_{i} d N_{i}(u) d x .
\end{aligned}
$$

Applying the stochastic Fubini theorem and exchanging the integral order, we obtain

$$
\begin{aligned}
\ln B\left(T_{f}, s\right)= & -\int_{T_{f}}^{s} f(0, x) d x \\
& -\int_{0}^{T_{f}} \int_{T_{f}}^{s} A(u, x-u) d x d u \\
& -\int_{0}^{T_{f}} \int_{T_{f}}^{s} \sigma(u, x-u) d x d_{u} \widetilde{W}(u, x-u) \\
& -\sum_{i=1}^{K} \int_{0}^{T_{f}} \int_{T_{f}}^{s} \gamma(u, x-u) d x a_{i} d N_{i}(u) .
\end{aligned}
$$

Therefore, it has

$$
\begin{aligned}
& F\left(t, T_{f}, s\right) \\
& =E_{t}^{\widetilde{P}}\left[B\left(T_{f}, s\right) \mid \Phi_{t}\right] \\
& =E_{t}^{\widetilde{P}}\left[\operatorname { e x p } \left(-\int_{T_{f}}^{s} f(0, x) d x-\int_{0}^{T_{f}} \int_{T_{f}}^{s} A(u, x-u) d x d u\right.\right. \\
& \quad-\int_{0}^{T_{f}} \int_{T_{f}}^{s} \sigma(u, x-u) d x d_{u} \widetilde{W}(u, x-u) \\
& \left.\left.\quad-\sum_{i=1}^{K} \int_{0}^{T_{f}} \int_{T_{f}}^{s} \gamma(u, x-u) d x a_{i} d N_{i}(u)\right) \mid \Phi_{t}\right] .
\end{aligned}
$$


Taking out what is known, we obtain

$$
\begin{aligned}
& F\left(t, T_{f}, s\right) \\
& =\exp \left(-\int_{T_{f}}^{s} f(0, x) d x\right. \\
& \quad-\int_{0}^{T_{f}} \int_{T_{f}}^{s} A(u, x-u) d x d u \\
& \quad-\int_{0}^{t} \int_{T_{f}}^{s} \sigma(u, x-u) d x d_{u} \widetilde{W}(u, x-u) \\
& \left.\quad-\sum_{i=1}^{K} \int_{0}^{t} \int_{T_{f}}^{s} \gamma(u, x-u) d x a_{i} d N_{i}(u)\right) \\
& \times E_{t}^{\widetilde{P}}\left[\exp \left(-\int_{t}^{T_{f}} \int_{T_{f}}^{s} \sigma(u, x-u) d x d_{u} \widetilde{W}(u, x-u)\right) \mid \Phi_{t}\right] \\
& \times E_{t}^{\widetilde{P}}\left[\exp \left(-\sum_{i=1}^{K} \int_{t}^{T_{f}} \int_{T_{f}}^{s} \gamma(u, x-u) d x a_{i} d N_{i}(u)\right) \mid \Phi_{t}\right] .
\end{aligned}
$$

Applying independent theorem in [10], we have

$$
\begin{aligned}
F\left(t, T_{f}, s\right) & =\exp \left[-\int_{T_{f}}^{s} f(0, x) d x\right. \\
& -\int_{0}^{T_{f}} \int_{T_{f}}^{s} A(u, x-u) d x d u \\
& -\int_{0}^{t} \int_{T_{f}}^{s} \sigma(u, x-u) d x d_{u} \widetilde{W}(u, x-u) \\
& \left.-\sum_{i=1}^{K} \int_{0}^{t} \int_{T_{f}}^{s} \gamma(u, x-u) d x a_{i} d N_{i}(u)\right] \\
\times E_{t}^{\widetilde{P}} & {\left[\exp \left(-\int_{t}^{T_{f}} \int_{T_{f}}^{s} \sigma(u, x-u) d x d_{u} \widetilde{W}(u, x-u)\right)\right] } \\
\times E_{t}^{\widetilde{P}} & {\left[\exp \left(-\sum_{i=1}^{K} \int_{t}^{T_{f}} \int_{T_{f}}^{s} \gamma(u, x-u) d x a_{i} d N_{i}(u)\right)\right], }
\end{aligned}
$$

where the first conditional expectation is

$$
\begin{aligned}
E_{t}^{\widetilde{P}}\left[\exp \left(-\int_{t}^{T_{f}} \int_{T_{f}}^{s} \sigma(u, x-u) d x d_{u} \widetilde{W}(u, x-u)\right)\right] \\
=\exp \left[\frac { 1 } { 2 } \int _ { t } ^ { T _ { f } } \left(\int_{T_{f}}^{s} \sigma(u, x-u) d x\right.\right. \\
\left.\left.\quad \times \int_{T_{f}}^{s} \sigma(u, y-u) c(x-u, y-u) d y\right) d u\right] .
\end{aligned}
$$

The conditional expectation of the increments of jump process is

$$
\begin{aligned}
& E_{t}^{\widetilde{P}}\left[\exp \left(-\sum_{i=1}^{K} \int_{t}^{T_{f}} \int_{T_{f}}^{s} \gamma(u, x-u) d x a_{i} d N_{i}(u)\right)\right] \\
& \quad=\exp \left[\sum_{i=1}^{K} \int_{t}^{T_{f}} \widetilde{\lambda_{i}}\left[\exp \left(-a_{i} \int_{T_{f}}^{s} \gamma(u, x-u) d x\right)-1\right] d u\right] .
\end{aligned}
$$

Thus, the price of futures is

$$
\begin{aligned}
F\left(t, T_{f}, s\right) & =\exp \left[-\int_{T_{f}}^{s} f(0, x) d x-\int_{0}^{T_{f}} \int_{T_{f}}^{s} A(u, x-u) d x d u\right. \\
& -\int_{0}^{t} \int_{T_{f}}^{s} \sigma(u, x-u) d x d_{u} \widetilde{W}(u, x-u) \\
& -\sum_{i=1}^{K} \int_{0}^{t} \int_{T_{f}}^{s} \gamma(u, x-u) d x a_{i} d N_{i}(u) \\
& +\frac{1}{2} \int_{t}^{T_{f}} \int_{T_{f}}^{s} \sigma(u, x-u) \\
& \left.\times \int_{T_{f}}^{s} \sigma(u, y-u) c(x-u, y-u) d y d x d u\right] \\
\times \exp & {\left[\sum_{i=1}^{K} \int_{t}^{T_{f}} \tilde{\lambda_{i}}\left[\exp \left(-a_{i} \int_{T_{f}}^{s} \gamma(u, x-u) d x\right)-1\right] d u\right] . }
\end{aligned}
$$

Rearranging (50), we obtain the futures price expressed as (41). It completes the proof.

\section{Acknowledgments}

Thanks are due to the referees whose meaningful suggestions are very helpful to revise the paper. This work is supported by the Fundamental Research Funds for the Central Universities (JBK130401).

\section{References}

[1] D. C. Heath, R. A. Jarrow, and A. Morton, "Bond pricing and the term structure of interest rates: a discrete time approximitation," Journal of Financial and Quantitative Analysis, vol. 25, no. 4, pp. 419-440, 1990.

[2] D. C. Heath, R. A. Jarrow, and A. Morton, "Bond pricing and the term structure of interest rates: a new methodology for contingent claims valuation," Econometrica, vol. 60, no. 60, pp. 77-105, 1992.

[3] D. P. Kennedy, "The term structure of interest rates as a Gaussian random field," Mathematical Finance, vol. 4, no. 3, pp. 247-258, 1994.

[4] D. P. Kennedy, "Characterizing Gaussian models of the term structure of interest rates," Mathemnatical Finance, vol. 7, no. 2, pp. 107-118, 1997. 
[5] R. S. Goldstein, "The term structure of interest rates as a random field," The Review of Financial Studies, vol. 13, no. 2, pp. 365-384, 2000.

[6] P. Santa-Clara and S. Didier, "The dynamics of the forward interest rate curve with stochastic string shocks," The Review of Financial Studies, vol. 14, no. 1, pp. 149-185, 2001.

[7] O. Vasicek, "An equilibrium characterization of the term structure," Journal of Financial Economics, vol. 5, pp. 177-188, 1977.

[8] J. Hull and A. White, "Pricing interest rate derivative securities," The Review of Financial Studies, vol. 3, no. 4, pp. 573-592, 1990.

[9] E. Eberlein, J. Jacod, and S. Raible, "Lévy term structure models: no-arbitrage and completeness," Finance and Stochastics, vol. 9, no. 1, pp. 67-88, 2005.

[10] S. E. Shreve, Stochastic Calculus for Finance II: Continuous-Time Models, Springer, New York, NY, US, 2004. 


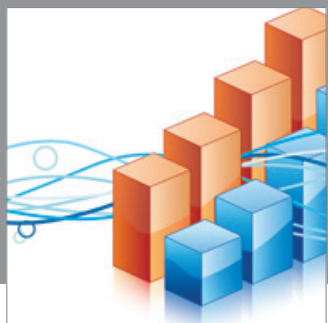

Advances in

Operations Research

mansans

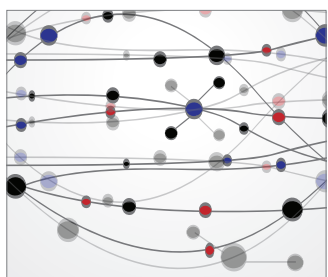

The Scientific World Journal
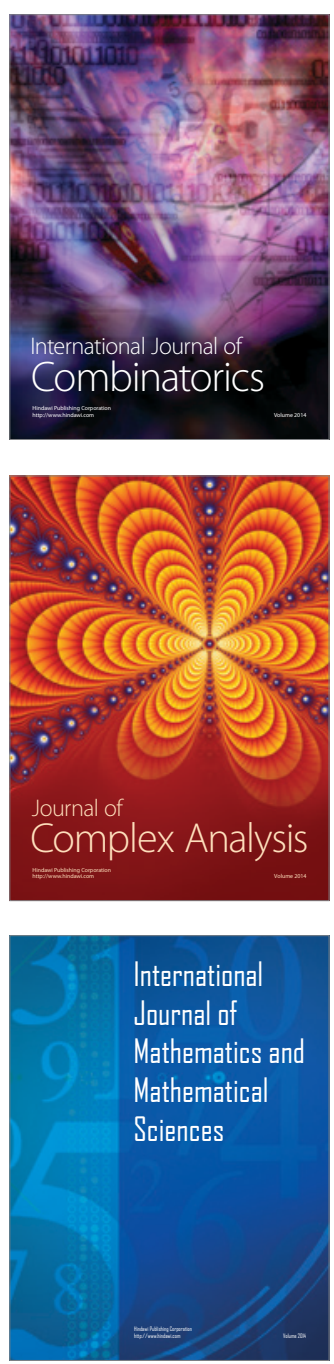
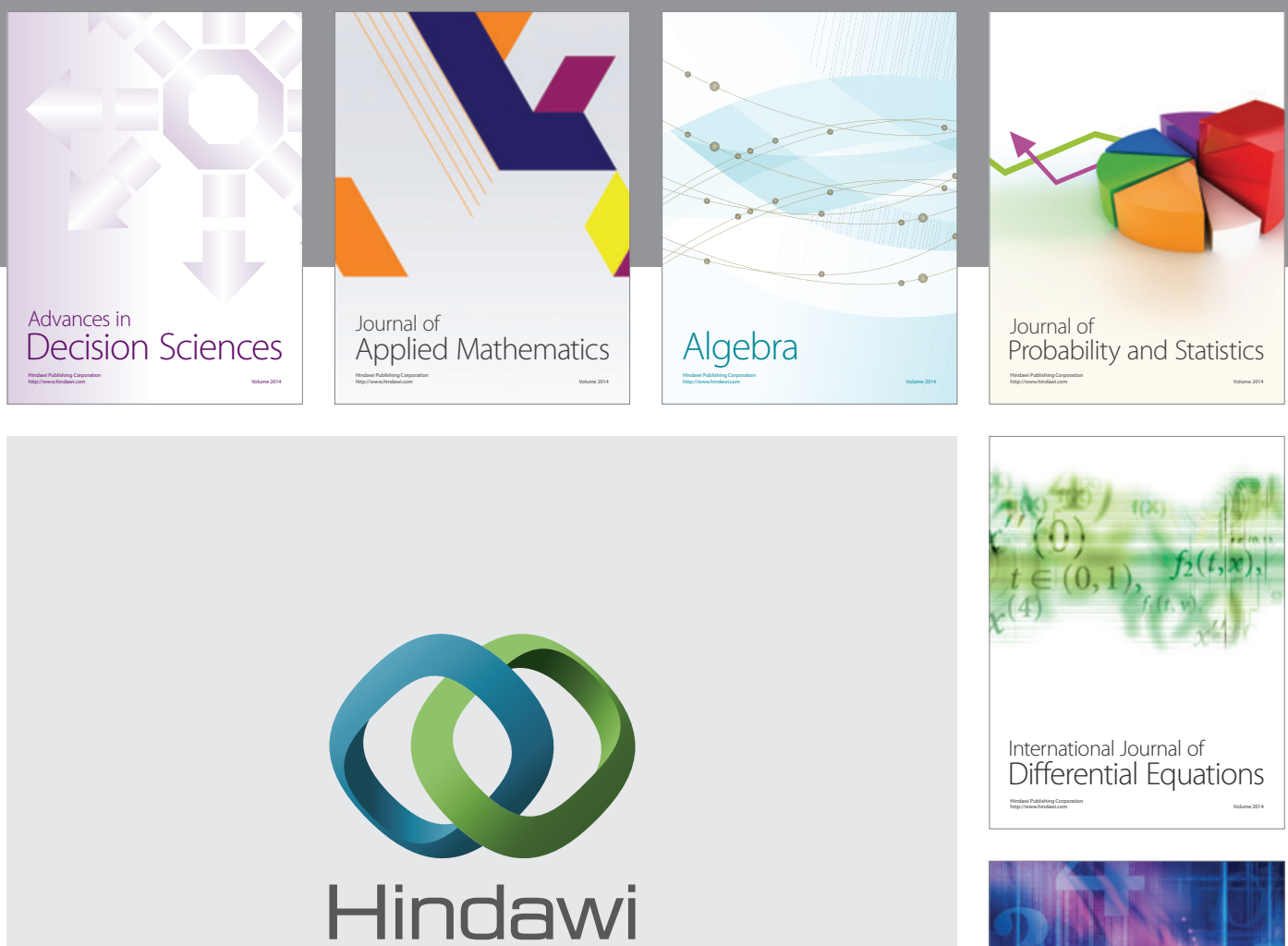

Submit your manuscripts at http://www.hindawi.com
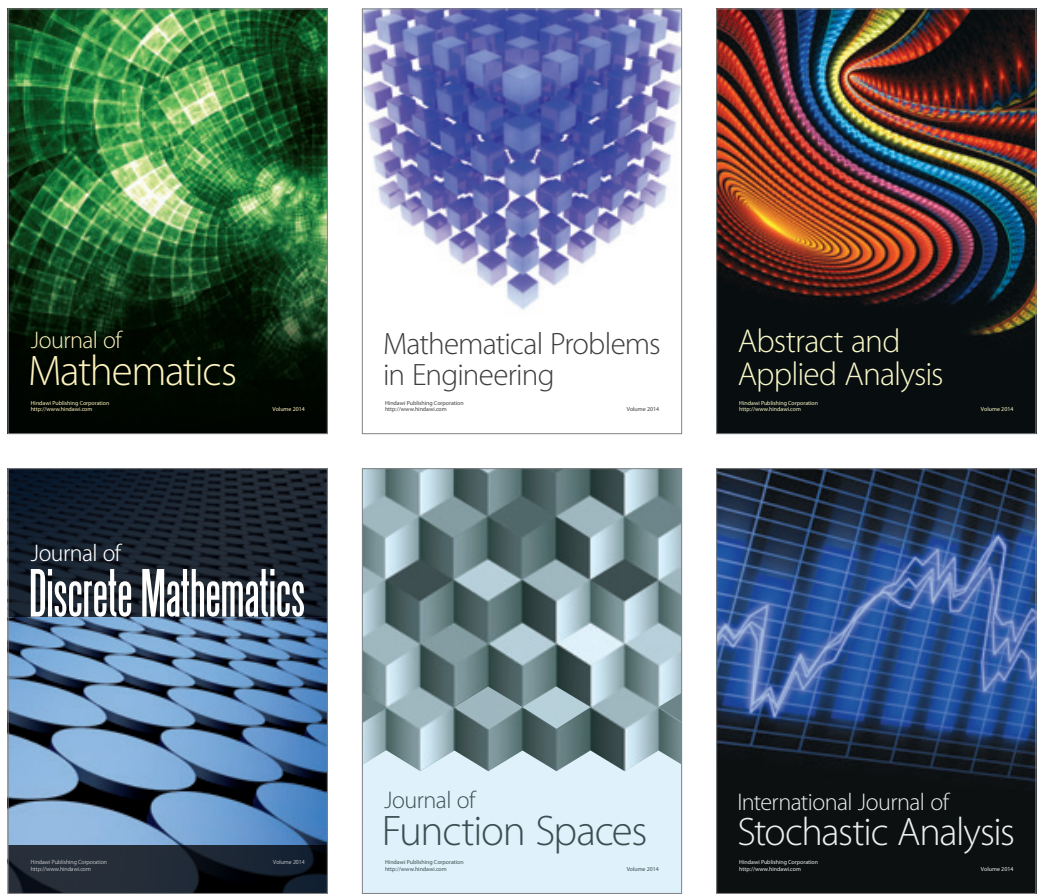

Journal of

Function Spaces

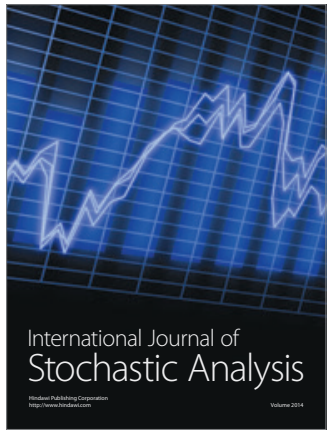

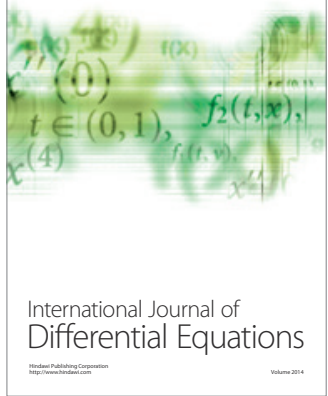
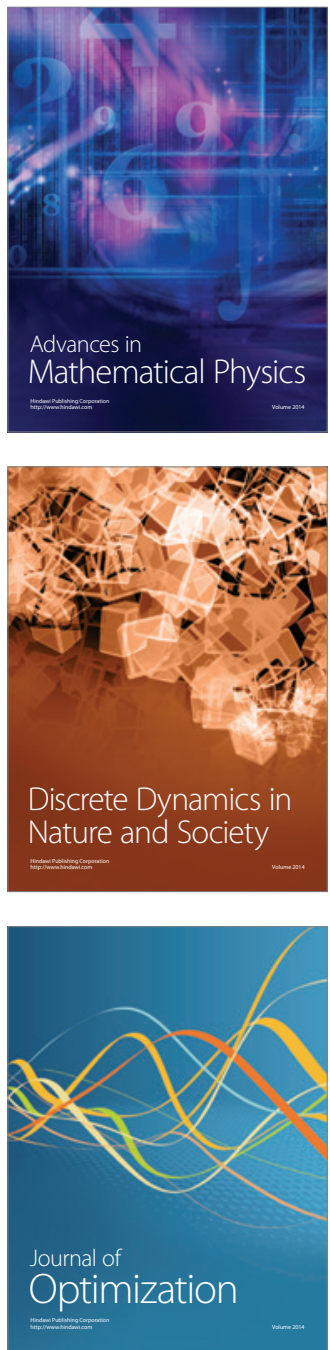